\title{
Prevention of hydrocephalus shunt catheter colonisation in vitro by impregnation with antimicrobials
}

\author{
R BAYSTON,* N GROVE, $\dagger$ J SIEGEL, $\ddagger$ D LAWELLIN,§ S BARSHAM* \\ From the Department of Paediatric Surgery, Institute of Child Health, London, UK*, Colorado Biomedical Inc, \\ Denver; $\dagger$ Codman and Shutleft Inc, Randolf; $\ddagger$ and the Kempe Research Center, Children's Hospital, Denver, $\S$ \\ USA
}

SUMMARY A process is described by which hydrocephalus shunt catheters can be impregnated with antimicrobials. The processed catheters showed antimicrobial activity at their surfaces for long periods and could be sterilised by autoclaving. When tested in vitro in a model of catheter colonisation using large challenge doses of Staphylococcus epidermidis and prolonged perfusion, some antimicrobials failed to protect against colonisation whereas others protected against one or two challenges. A combination of rifampicin and clindamycin gave best results, protecting against three successive challenges over a 28 day perfusion period. Resistant organisms did not develop. The process is likely to be useful in prevention of hydrocephalus shunt infection.

The reported incidence of hydrocephalus shunt infections ranges from $1 \%$ to $39 \%$ of operations ${ }^{12}$ with a national average of about $10 \%$. In most cases the causative organisms originate on the patient's skin and gain access to the shunt system during its insertion or revision. ${ }^{3}$ There is an additional slight risk later due to needling of the shunt for pressure measurement or fluid aspiration.

The organisms most commonly involved are gram positive, with coagulase negative staphylococci predominating.

Various attempts to prevent shunt infections have been made, including modifications of technique ${ }^{13}$ and the use of prophylactic antibiotics, ${ }^{4}$ and in some cases the rate of infection has diminished. However, shunt infection is still a major problem in most centres, causing considerable morbidity and some mortality as well as consuming resources extensively.

Similar infections are encountered with most implantable devices, including cardiac valves and patches, central venous catheters, vascular grafts and pacemaker capsules. Attempts have been made to make such implants resistant to infection by various methods involving direct or indirect bonding of antimicrobials to the implant surface, ${ }^{s-10}$ with variable

Address for reprint requests: Dr R Bayston, Department of Paediatric Surgery, Institute of Child Health, 30 Guilford Street, London WCIN IEH.

Received 29 July 1988 and in revised form 10 November 1988. Accepted 18 November 1988 success. Using a different approach, Kingston et al ${ }^{11}$ mixed a disinfectant into three implantable polymers before processing. With one exception, ${ }^{9}$ the processes have involved polymers such as polytetra-fluoroethylene (PTFE), polyethylene and dacron, which are used mainly for vascular grafts, but they have not been used with silicone elastomer. Varieties of this material are widely used for central venous catheters, pacemaker capsules, artificial urinary sphincters and small joint replacements as well as for hydrocephalus shunts. Several methods have been developed and assessed for incorporating antimicrobials into silicone elastomer ${ }^{12-15}$ and one has been found suitable for use after the manufacturing process has been completed. ${ }^{1415}$ The process results in complete incorporation of the antimicrobial into the elastomer matrix without discernible effect on its appearance on electron microscopy or on its mechanical properties, while conferring long-lasting antimicrobial activity at the surface of the implant, which may be autoclaved without significant loss of activity. Evaluation in vitro of the protection against bacterial colonisation conferred by this process is reported here.

\section{Methods}

Incorporation of antimicrobials

The antimicrobials studied were rifampicin, trimethoprim, spiramycin [Sigma Chemical Company UK), clindamycin hydrochloride (Upjohn Ltd., UK), and diethanolamine fusidate (Leo Pharmaceutical Products, Denmark). Solu- 
tions of the antimicrobials, singly or in combinations, were made in chloroform to give a concentration of each $(w / v)$ of $0 \cdot 2 \%$. No adjustment was made for potency. Supplementary studies were carried out with rifampicin and clindamycin hydrochloride $(0.025 \%, 0.05 \%$ and $0.1 \%)$ and rifampicin and diethanolamine fusidate $(0 \cdot 1 \%)$ combinations. A total of nine catheters processed with rifampicin and clindamycin hydrochloride each in a $0.2 \%$ solution were tested.

For each experiment three $25 \mathrm{~cm}$ lengths of silicone shunt catheter (Dow Corning Corporation USA) were totally submerged in the antimicrobial solution, taking care to ensure filling of the tubing, for one hour at room temperature with shaking. The tubing was then removed, shaken to remove excess solution and rinsed and flushed in ethanol. After further shaking and blotting the catheters were airdried overnight at room temperature.

\section{Preliminary test for activity}

Plates of DST agar (Oxoid UK) which had been seeded with the Oxford strain of Staphylococcus aureus (NCTC 6571) were prepared and two wells, $1 \mathrm{~cm}$ diameter and $2 \mathrm{~mm}$ apart, were cut using a specially made punch. After autoclaving at $121^{\circ} \mathrm{C}$ for 20 minutes, three segments of each processed catheter, $0.5 \mathrm{~cm}$ long, were placed aseptically on the $2 \mathrm{~mm}$ bridge so that they lay at right angles to it (fig). The purpose of the technique is to avoid contact between the seeded agar and the cut surface of the tubing as this has given misleading results in the past. After incubation the diameters of the zones of inhibition at right angles to the long axis of the catheter segments were measured. Segments of unprocessed catheters and of catheters processed without antimicrobials served as controls.

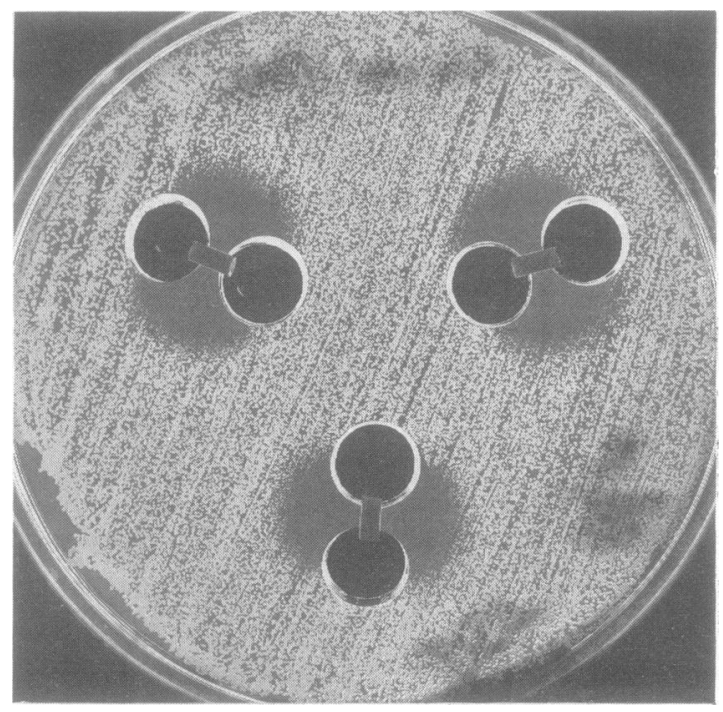

Fig Processed, autoclaved catheter segments screened for antimicrobial activity by the bridge plate method. After inoculation the diameters of the zones of inhibition are measured in millimetres at right-angles to the long axes of the catheter segments.

\section{In Vitro Challenge}

The model of catheter colonisation was that described previously. ${ }^{1617}$ Briefly, the test catheter is inserted into a controlled-environment chamber and autoclaved. The chamber is then connected to a heated water circuit and maintained at $37^{\circ} \mathrm{C}$ throughout. The catheter is perfused with nutrient fluid, in this case brain-heart infusion medium (Oxoid UK), at a controlled rate. All procedures are carried out using vigorous aseptic technique to avoid contamination.

The challenge organism was a strain of Staphylococcus epidermidis whose biochemical and other characteristics were known in detail, and which had been isolated from a colonised shunt. It was susceptible to all the antimicrobials used in the process. The challenge dose consisted of $1 \mathrm{ml}$ of an overnight culture of the organism in brain heart infusion, found by viable counting to contain $1 \times 10^{7}-1 \times 10^{8}$ colony-forming units per $\mathrm{ml}$. After injection of the challenge dose, the catheters were perfused at a rate of $10 \mathrm{ml}$ per hour. An unprocessed translucent catheter was included as a control to ensure that the challenge organism was capable of colonising, and all were tested in triplicate. A detailed description of the model and of the challenge and monitoring procedure is given elsewhere. ${ }^{17}$ The catheters were examined visually each day and samples of perfusion fluid were collected periodically for culture. If no colonisation was detected after 2 weeks' perfusion, a second challenge dose was given, with a second control catheter. Again, if the test catheters remained free of colonisation after a further 2 weeks' perfusion, a third challenge was made with a third control catheter, followed again by monitoring and perfusion for 2 weeks. Any catheters remaining apparently uncolonised were then removed from the system and examined thoroughly for bacteria using a flushing technique. ${ }^{18}$ Any organisms isolated from the catheters at any stage were carefully identified and their characteristics compared to those of the challenge organisms in order to discount contamination. They were also tested for susceptibility to the antimicrobials present in the catheter from which they had been recovered.

\section{Results}

The combination of trimethoprim and clindamycin hydrochloride could not be tested owing to chemical incompatibility of the two antimicrobials in the impregnation process.

Preliminary testing of all processed, autoclaved catheters on bridge plates showed antimicrobial activity (fig). Segments not containing antimicrobials showed no activity.

Results of challenge of catheters which had been processed using a concentration of each antimicrobial of $0.2 \% \mathrm{w} / \mathrm{v}$ are shown in table 1 . The combinations of diethanolamine fusidate with trimethoprim and one of the three catheters containing rifampicin with spiramycin failed to protect against the first challenge, as did all the catheters containing single antimicrobials with the exception of rifampicin. The remaining two catheters with rifampicin and spiramycin, those with rifampicin and trimethoprim, and those with rifam- 
picin alone withstood the first challenge but failed to protect against the second. Only those catheters containing rifampicin with clindamycin hydrochloride withstood all three challenges.

The results of further studies using different concentrations of rifampicin and clindamycin are shown in table 2 . Those catheters processed with less than $0 \cdot 1 \%$ of each antimicrobial failed to withstand the first challenge. Of the six catheters processed with $0 \cdot 1 \%$, five withstood the first two challenges but became colonised after the third. Impregnation with diethanolamine fusidate and rifampicin, both at $0.1 \%$, resulted in colonisation of four catheters on first challenge and the remaining two on second challenge. Once again, only those processed with $0.2 \%$ of rifampicin and clindamycin hydrochloride withstood all three challenges.

None of the organisms isolated from the catheters which became colonised showed resistance to the antimicrobials used, all minimum inhibitory concentrations (MIC) remaining unchanged compared to those of the inocula.

\section{Discussion}

The antimicrobials were chosen according to several criteria. The first was that they should be capable of molecular migration through crosslinked silicone elastomer, an index of this being solubility in chloroform. ${ }^{19}$ The second criterion was that they should be active against most strains of staphylococci, and the third was that they should have been administered systemically in humans without known significant risk of hypersensitivity or toxicity. The fourth

Table 1 Resistance to colonisation of processed catheters after three consecutive challenges fourteen days apart, during constant perfusion

\begin{tabular}{lllll}
\hline & \multicolumn{2}{l}{ Challenge } & $\begin{array}{l}\text { Number of } \\
\text { catheters } \\
\text { tested }\end{array}$ \\
\cline { 2 - 5 } Antimicrobial (all $0 \cdot 2 \%$ )* & 1 & 2 & 3 & 6 \\
\hline Rifampicin & - & $+(6)$ & ND & 3 \\
Trimethoprim & $+(3)$ & ND & ND & 3 \\
Clindamycin & $+(3)$ & ND & ND & 3 \\
Spiramycin & $+(3)$ & ND & ND & 3 \\
Fusidate & $+(3)$ & ND & ND & 3 \\
Trimethoprim + fusidate & $+(3)$ & ND & ND & 3 \\
Trimethoprim + rifampicin & - & $+(3)$ & ND & 3 \\
Spiramycin + rifampicin & $+(1)$ & $+(2)$ & ND & 3 \\
Rifampicin + fusidate & $+(2)$ & $+(4)$ & ND & 6 \\
Rifampicin + clindamycin & - & - & - & 3 \\
\hline
\end{tabular}

Clindamycin = clindamycin hydrochloride

Fusidate $=$ diethanolamine fusidate

$-=$ No colonisation

$+=$ Colonised

ND $=$ Not done

Figures in parentheses are the number of catheters becoming colonised on each occasion.

*The concentration of each antimicrobial in the impregnation fluid.
Table 2 Resistance to colonisation of catheters processed with different concentrations of antimicrobials after three consecutive challenges fourteen days apart, during constant perfusion

\begin{tabular}{|c|c|c|c|c|}
\hline \multirow{2}{*}{$\begin{array}{l}\text { Antimicrobial and } \\
\text { concentration* }\end{array}$} & \multicolumn{3}{|c|}{ Challenge } & \multirow{2}{*}{$\begin{array}{l}\text { Number of } \\
\text { catheters } \\
\text { tested }\end{array}$} \\
\hline & 1 & 2 & 3 & \\
\hline \multirow{5}{*}{$\begin{array}{l}\text { Rifampicin } 0.2 \% \\
\text { + clindamycin } 0.2 \% \\
\text { Rifampicin } 0.1 \% \\
\text { + clindamycin } 0.1 \% \\
\text { Rifampicin } 0.05 \% \\
\text { + clindamycin } 0.05 \% \\
\text { Rifampicin } 0.025 \% \\
\text { + clindamycin } 0.025 \% \\
\text { Rifampicin } 0.1 \% \\
\text { + fusidate } 0.1 \%\end{array}$} & - & - & - & 6 \\
\hline & - & $+(1)$ & $+(5)$ & 6 \\
\hline & $+(3)$ & ND & ND & 3 \\
\hline & $+(3)$ & ND & ND & 3 \\
\hline & $+(4)$ & $+(2)$ & ND & 6 \\
\hline
\end{tabular}

Abbreviations as in table 1 .

criterion was that they should be sufficiently stable to allow sterilisation by autoclaving. Many antimicrobials were excluded on one or more of these grounds.

Hypersensitivity caused by the antimicrobials used here has been reported only occasionally, and toxic side-effects have been associated with prolonged therapeutic use or high dosage. The total concentration of antimicrobial in a processed shunt has been found to be less than a single therapeutic dose, and this is released slowly over several weeks, making major organ toxicity such as hepatic dysfunction very unlikely. A further possibility is the local accumulation of antimicrobial in the tissues immediately adjacent to the catheter with local toxicity or irritation. However, animal toxicological studies have not shown any such local or systemic irritation or toxicity in a variety of tissues including neonatal brain (Bayston et al, unpublished). When processed autoclaved catheters were incubated on bridge plates they showed acceptable activity. This test serves as a preliminary screen prior to perfusion and challenge tests. While the method tests the external surface of the catheter rather than the luminal surface, the antimicrobial activity at each surface is similar after this process and the screening test gives a useful indication of activity at the luminal surface.

The drug combination which appeared to give best results in the perfusion test was rifampicin with clindamycin hydrochloride, both at a concentration of $0.2 \% \mathrm{w} / \mathrm{v}$ in the impregnation fluid. Attempts to decrease the concentration of the antimicrobials led to failure of protection for the target period, as shown in table 2 . The 28 day period of protection in vitro was chosen in order to ensure protection over the operative and immediate postoperative period and in terms of the main period of risk of infection in shunts is probably excessive, but the period of risk in other 
implants sometimes extends beyond this. Also, no protection by this process would be expected against shunt infection arising later from causes such as visceral perforation.

Almost all coagulase negative staphylococci isolated from colonised shunts are susceptible to rifampicin, and the majority are susceptible to clindamycin hydrochloride.$^{20}$ When rifampicin is used alone therapeutically, resistance develops rapidly ${ }^{21}$ and this is the main reason for combining it with another antimicrobial. There may be other benefits from the use of the chosen combination of rifampicin and clindamycin hydrochloride, as combinations with other antimicrobials were shown to be inferior. For example, it has been suggested that clindamycin prevents attachment of organisms to implants, ${ }^{22}$ though this antimicrobial alone had no effect in our study.

No resistant organisms were found among those isolated from catheters which became colonised, suggesting that failure to protect was probably due to inadequate antimicrobial activity at the surface of the catheter. In those catheters where protection failed at the time of second or third challenge, implying that the adequate antimicrobial activity present on first challenge had waned, colonising organisms still had the same MIC as the inoculum. This shows that resistance does not develop even when the antimicrobials are present in sub-lethal amounts. The finding also suggests that development of resistance in clinical use will not be a problem. The hydrocephalus shunt is not continuously exposed to skin flora as in the central venous catheter, where resistance could develop around the entry site. In addition, animal studies indicate that tissue, plasma and urine levels of antimicrobials are likely to be far too low to affect the normal flora of the skin, alimentary tract and mucous membranes (Bayston et al, unpublished).

The in vitro model of catheter colonisation is intentionally concerned with colonisation of the luminal surface. Very soon after silicone elastomer implants are inserted into the body, their surfaces which are in contact with blood or tissue become coated with fibronectin, fibrin, albumen and other materials which significantly change the nature of the surface of the implant. ${ }^{23}$ However, such biocoatings are not usually seen on the luminal surfaces of hydrocephalus shunts. In addition, luminal colonisation, usually by coagulase negative staphylococci, is much more common than external infection which constitutes a wound infection around a foreign body and is usually due to Staph. aureus or gram negative bacteria. The impregnation process is intended to protect against luminal colonisation though it may in some cases also reduce the risk of external infections.

The size of the bacterial challenge is several orders of magnitude higher than that to be expected clinically $y^{25}$ but is similar to that used in animal challenge studies and in our hands was found to be necessary to ensure consistent colonisation of control catheters in vitro. Even so, the catheters impregnated with rifampicin and clindamycin hydrochloride using a $0.2 \%$ solution were able to withstand three successive challenges of this size.

Several attempts have been made by others to confer antibacterial activity on implants. Early attempts involved the bonding of penicillin or oxacillin to PTFE using benzalkonium chloride or tridodecylmethylammonium chloride (TDMAC). ${ }^{5-9}$ This method has the disadvantage of short duration of activity, not exceeding a few days. Also, catheters with bonded antimicrobial cannot be sterilised $^{8}$ and would therefore be at risk of contamination from gram negative bacteria, especially pseudomonas, and fungi. The coating process is not likely to be suitable for hydrocephalus shunts as benzalkonium chloride and TDMAC cannot be used in contact with nervous tissue, and the coating might have a deleterious effect on the function of valve mechanisms which are known to be susceptible to changes in surface properties of the elastomer. Similarly, the coating method described by Modak et al ${ }^{10}$ for PTFE grafts has short duration, cannot be sterilised and uses potentially neurotoxic materials.

The method described by Kingston et al, ${ }^{11}$ in which the disinfectant trichlorohydroxydiphenylether (Irgasan DP 300, Ciba-Geigy) was mixed into implantable thermoplastics before moulding, gave very long-lasting activity but once again the agent has not been tested for neurotoxicity by the authors. Manufacturer's data on toxicology suggest that the substance would be irritant or toxic to some tissues if implanted. In addition the concentrations required in order to give the desired activity would almost certainly have deleterious mechanical effects on silicone valve mechanisms. ${ }^{14}$ The process described here using rifampicin and clindamycin hydrochloride has been shown to result in intimate incorporation of the antimicrobials in the matrix of the silicone elastomer, ${ }^{19}$ with the absence of particles or cavitations. The mixing of particles into the matrix would also be likely to lead to significant changes in mechanical properties if they were to be removed by aqueous dissolution after implantation, leaving a spongiform texture.

Catheters processed with rifampicin and clindamycin hydrochloride using a $\mathbf{0} \cdot 2 \%$ solution have been shown to resist three consecutive massive challenges over 28 days of constant perfusion. While resistance of the first challenge only, as was found with these two antimicrobials at $0 \cdot 1 \%$ as well as with trimethoprim with rifampicin and rifampicin alone, would probably 
be beneficial in covering the period of risk in most cases, the prolonged period of activity should also provide protection at early revisions or during postoperative needling for pressure studies or fluid aspiration. In addition, shunts resistant to colonisation for more than just a few days might be useful in patients with hydrocephalus who have shunt-associated ventriculitis. The recommended treatment involves shunt removal and a period of external ventricular drainage with antimicrobial therapy before reshunting. There is a considerable risk of secondary infection during external drainage, which also provides imperfect control of cerebrospinal fluid pressure in many cases. Here, external ventricular drainage could perhaps be dispensed with, and a new processed shunt inserted immediately on removal of the colonised one without risk of colonisation of the new one, while providing regulated internal control of intracranial pressure and eliminating the need for a further surgical procedure. However, the effects of constant exposure of the processed catheter similar to that expected during the first 24-48 hours in this clinical situation need to be evaluated in vitro.

Thanks are due to the Essex, Wigan and Leigh and Norwich branches of the Association for Spina Bifida and Hydrocephalus and the Wade Fund for financial support and to Mrs Roz Sullivan for typing the manuscript.

\section{References}

1 Tabara Z, Forrest DM. Colonisation of CSF Shunts: Preventive Measures. Z Kinderchir 1982;37:156-8.

2 Wald S, McLaurin RL. Cerebrospinal Fluid Antibiotic Levels during treatment of Shunt Infections. $J$ Neurosurg 1980;52: 41-6.

3 Fitzgerald R, Connelly B. An operative technique to reduce valve colonisation. Z Kinderchir 1984;39 Suppl II:107-9.

4 Haines SJ. Systemic Antibiotic Prophylaxis in Neurological Surgery. Neurosurg 1980;6:355-61.

5 Jagpal R, Greco RS. Studies of a graphite-benzalkonium-oxacillin surface. Am Surg 1979;45:774-9.

6 Moore WS, Chvapil M, Seiffert G, Keown K. Development of an infection-resistant vascular prosthesis. Arch Surg 1981; 116:1403-7.

7 Harvey RA, Greco RS. The noncovalent bonding of antibiotics to a polytetrafluoroethylene-benzalkonium graft. Am Surg 1981;
194:642-7.

8 Donetz AP, Harvey RA, Greco RS. Stability of antibiotics bound to polytetrafluoroethylene with cationic surfactants. $J$ Clin Microbiol 1984;19:1-3.

9 Trooskin SZ, Donetz AP, Harvey RA, Greco RS. Prevention of catheter sepsis by antibiotic bonding. Surgery 1985;97:547-51.

10 Modak SM, Sampath L, Fox CL, Benvenisty A, Nowygrod R, Reemstmau K. A new method for the direct incorporation of antibiotic in prosthetic vascular grafts. Surg Gynecol Obstet 1987;164:143-7.

11 Kingston D, Seal DV, Hill ID. Self-disinfecting plastics for intravenous catheters and prosthetic inserts. $\mathrm{J} \mathrm{Hyg}$ 1986;96:185-98.

12 Bayston R. Preliminary Studies on the impregnation of silastic elastomers with antimicrobial substances. Devel Med Child Neurol 1975;18 Suppl 37:50-4.

13 Bayston R. The antibacterial effects of impregnated silastic and its possible applications in surgery. $J$ Ped Surg 1977;12:55-61.

14 Bayston R. The effect of antibiotic impregnation on the function of slit valves used to control hydrocephalus. $Z$ Kinderchir 1980;31:353-9.

15 Bayston R, Milner RDG. Antimicrobial activity of silicone rubber used in hydrocephalus shunts, after impregnation with antimicrobial substances. J Clin Pathol 1981;34:1057-62.

16 Bayston R, Adroyewski V, Barsham S. Use of an in vitro model to study eradication of catheter colonisation by Staphylococcus epidermidis. $J$ Infect 1988;16:141-6.

17 Bayston R, Barsham S. Catheter colonisation: A laboratory model suitable for aetiological, therapeutic and preventive studies. Med Lab Sci 1988;45:235-9.

18 Bayston R, Leung TSM, Wilkins BM, Hodges B. Bacteriological examination of removed cerebrospinal fluid shunts. $J$ Clin Pathol 1983;36:987-90.

19 Bayston R. The Impregnation of medical silicone elastomers and acrylic bone cements with antimicrobial drugs. PhD Thesis, University of Sheffield, 1979.

20 Archer GL. Antimicrobial Susceptibility and selection of resistance among Staphylococcus epidermidis isolates recovered from patients with infections of indwelling foreign devices. Antimicrob Ag Chemother 1978;14:353-9.

21 Binda G, Domenichini E, Gottardi A, Orlandi B, Ortelli E, Pacini B, Fowst G. Rifampicin, a general review. Arzneim Forsch 1971;21:1907-77.

22 Pfaller M, Davenport D, Bale M, Barrett M, Koontz F, Masanari $M$. Development of a semiquantitative test for slime production: Application to the study of sub-inhibitory effects of antistaphylococcal agents. Abstract No L-43. In: Abstract of the Annual Meeting of the American Society for Microbiology, Washington DC, American Society for Microbiology 1986.

23 Dankert J, Hogt A, Feizen J. Biomedical Polymers: Bacterial Adhesion, colonisation and infection. CRC Critical Reviews in Biocompatibility 1986;2:219-301.

24 Raahave D. Bacterial Density in operation wounds. Acta Chir Scand 1974;140:585-93.

25 Benediktsdottir E, Hambraeus A. Isolation of anaerobic and aerobic bacteria from clean surgical wounds: an experimental and clinical study. J Hosp Infect 1983;4:141-8. 